\title{
The Nozzle Structure Design and Analysis for Continuous Carbon Fiber Composite 3D Printing
}

\author{
Fan Zhang ${ }^{1, a}$, Guofeng $\mathrm{Ma}^{1, \mathrm{~b}}$ and Yuegang $\operatorname{Tan}^{1, \mathrm{c}}$ \\ ${ }^{1}$ School of Mechanical and Electrical Engineering, Wuhan University of Technology, Wuhan, \\ 430070, China \\ afan_dx0202@163.com, b940173793@qq.com, cygtan@whut.edu.cn
}

\begin{abstract}
Keywords: Continuous carbon fiber composite, 3D printing, Nozzle structure, Thermodynamic simulation
\end{abstract}

Abstract: The 3D printing technology for continuous carbon fiber composite can combine the advantages of high mechanical properties and unlimited model structure complexity to complete the rapid prototyping. Because of the continuity characters of the carbon fiber composite, if the nozzle temperature is too high, the nozzle will be blocked due to material excessive softening. But if the temperature is low, it can't pile up in sequential layers. For this problem, the 3D printing nozzle structure was designed and optimized by the thermodynamic simulation, also, the relations between the nozzle structure and temperature field distribution was revealed. The rationality of nozzle design is proved by continuous carbon fiber 3D printing experiment.

\section{Introduction}

The carbon fiber composite has been used in the areas of aerospace, ultralight automobile, petrochemical industry et al. which is called the strategic material. 3D printing technology used in carbon fiber compoiste prototype manufacturing can bring into full play both excellent features of light weight, high strength, integrated molding, and unlimited model structure complexity[1,2]. The traditional carbon fiber composite complex forming process can be improved and the no-metallic functional mechanical components can be realized by 3D printing technology[3,4].

The printer nozzle is one of the most important components for rapid prototyping. In the printing process, if the nozzle temperature is lower than the normal range, the incomplete softening thermoplastic composite will lead poor cohesiveness between the different lines and layers of the molding part. If the nozzle temperature is higher than the normal value, the over softening materials before the extrusion nozzle will cause the jamming problem. So, the optimized design of nozzle structure with reasonable temperature field distribution is the key factor for high quality continuous carbon fiber composite 3D printing.

For the material continuity and anisotropy characters, there are few research results in continuous carbon fiber composite 3D printing. In the Toyohashi University of Technology, after several lays of thermoplastic material were printed, the continuous carbon composite was arranged in the middle, then the 3D printing was continued to complete the forming process. By this way, the effect of continuous carbon fiber on thermoplastic material property was tested [5]. In Xi' an Jiao Tong University and Nanjing University of Aeronautics \& Astronautics of China, the Polylactice Acid ( PLA) and continuous carbon fiber material were printed together by Fused Deposition Modeling (FDM) 3D printing technology. The continuous carbon fiber was stuffed into the printer nozzle passively relying on the PLA melting extrusion pressure to achieve the simple path model printing. Also, the modeling performance between PLA and enhanced continuous carbon fiber composite was compared [6-8]. In the Tokyo University of Science, the resin soaked carbon fiber was used as the printing material to print the test sample. The experimental results show that this method can greatly enhance the strength of the product. It is concluded that the increasing adhesive force between the thermoplastic material and carbon fiber and the more number of fiber tows, the modeling performance can be further enhanced. These studies are all in the initial experimental stage basically, the optimized molding method, the reasonable molding conditions, and the relationship of different process 
parameters are need to be further studied to enhance the 3D printing quality of continuous carbon fiber composite.

In this paper, the 3D printing process of the continuous carbon fiber composite is introduced first. Then, according to the materials characters, the printing nozzle structure is designed and the law of temperature field distribution is simulated and explored. Finally, the correctness of the design is verified by the 3D printing experiment of continuous carbon fiber composite.

\section{D printing forming process of continuous carbon fiber composite}

The forming principle for carbon fiber cmoposite 3D printing is shown in Figure 2.1.

1) The continuous carbon fiber is pretreated by the thermoplastic polymer materials.

2) The raw material is sent to the feeding device and the thermoplastic material is heated to a molten state.

3) The path instruction is carried out by the control system.

4) The $3 \mathrm{D}$ objects are printed layer by layer.

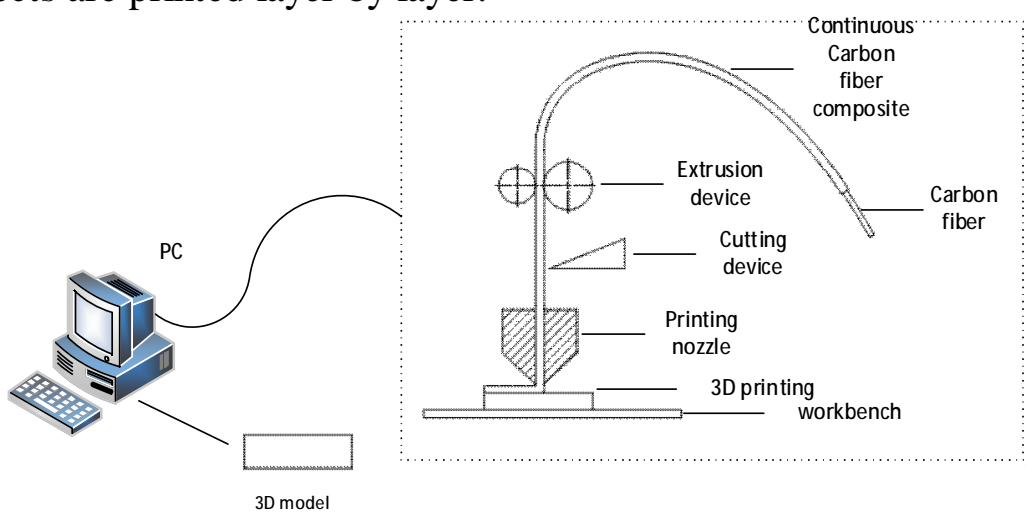

Figure 2.13D printing forming process of continuous carbon fiber composite

According to the different part functions of the 3D printing extrusion nozzle, it can be divided into five parts: positioning area, feeding area, cutting area, guiding area, fusing area and forming area, as shown in Figure 2.2.

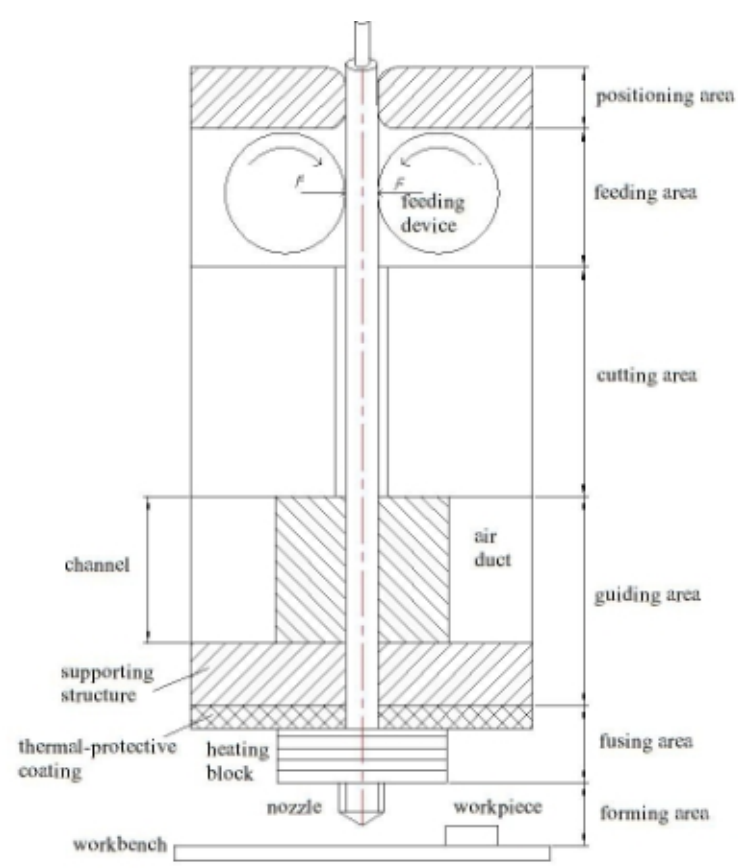

Figure 2.2 The functional areas of the 3D printing nozzle 


\section{Nozzle device design of the continuous carbon fiber composite 3D printing}

Nozzle device structure. According to the characters of the material and the functions of the extrusion nozzle device, the nozzle structure is shown in Figure 3.1.

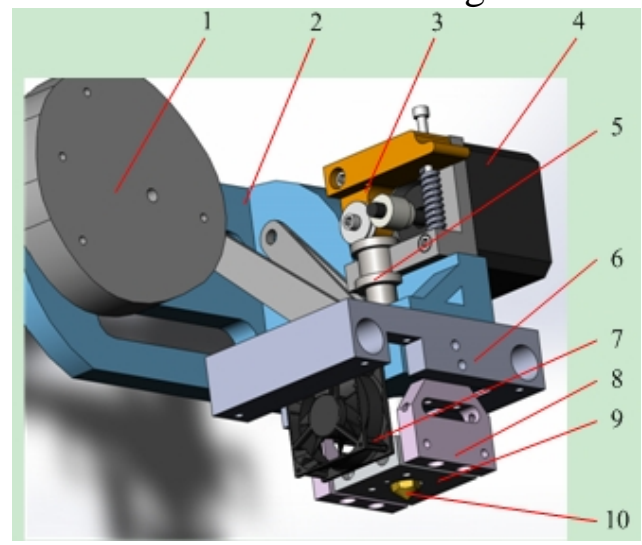

Figure 3.1 Nozzle structure of the carbon fiber composite 3D printing

1.cutting device, 2.fixing structure, 3.extrusion structure, 4.extrusion motor, 5.pipewok, 6.movement slider, 7.cooling fan, 8.fixed support, 9.heating block, 10.printing nozzle

The stepper motor which is driven by the printing signal drives the extrusion mechanism to work. In the extrusion mechanism, the carbon fiber composite material is preloaded by the spring and clamped between the two feed wheels. The feed wheels make the $U$ type bearing rotation and the material is feed to the throat channel of the guiding area relying on the friction movement. The material is transported in the throat channel by the piston action, melt by the heating block and extruded by the printing nozzle.

\section{Heating and cooling system of the nozzle mechanism}

Heating and cooling structure. The matrix material of the continuous carbon fiber inside of the nozzle will change from the solid state to the molten state. Two electric heating robs are used to provide energy to realize the rapid melting in the nozzle. The heating nozzle structure is shown in Figure 3.2. At the same time, the heat dissipation problem also has to be resolved to avoid the throat channel blocked because of the softened matrix material. The heat conduction is reduced by setting the heat insulating layer between the heating block and the fixed structure as shown in Figure 3.3.

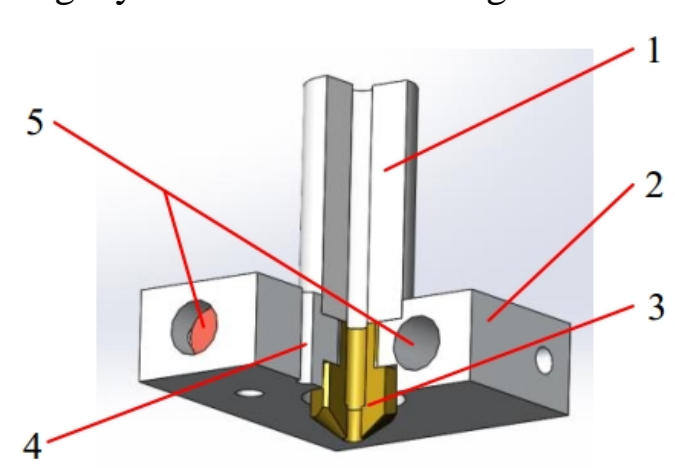

Figure 3.2 heating structure

1.throat channel 2.heating block 3.printing nozzle 4.temperature sensor 5.heating rod

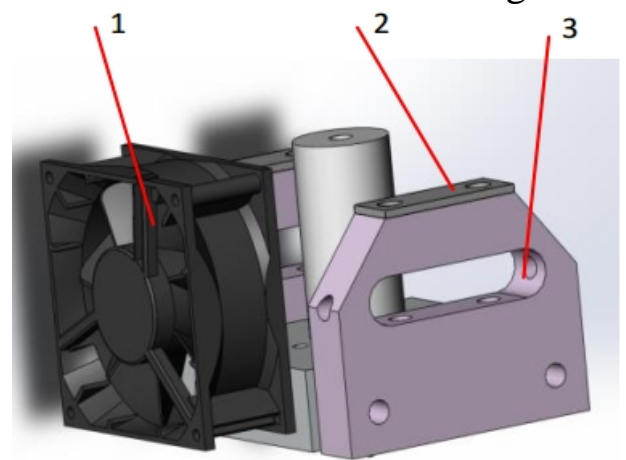

Figure 3.3 heating dissipation structure 1.heating dissipation fan 2.heating insulating layer 3.fixed structure

\section{Temperature field distribution simulation and structure optimization}

Temperature distribution simulation. The finite element mesh of the nozzle device is divided by the intelligent structure mesh method. According to the requirement of the nozzle temperature, the heating block temperature is 200 degrees Celsius; the motor surface temperature is 25 degrees Celsius. The heat transfer mode is the contact surface of the internal parts and components. The electric fan is 
used to realize the heating convection cooling. The fan speed is $5 \mathrm{~m} / \mathrm{s}$, the convection coefficient is 100 . The nozzle, fixed structure, heating block and motor surface are selected as the heat radiation surface. The radiation coefficient is 0.3. The heating field distribution is shown in Figure 3.4 and 3.5.

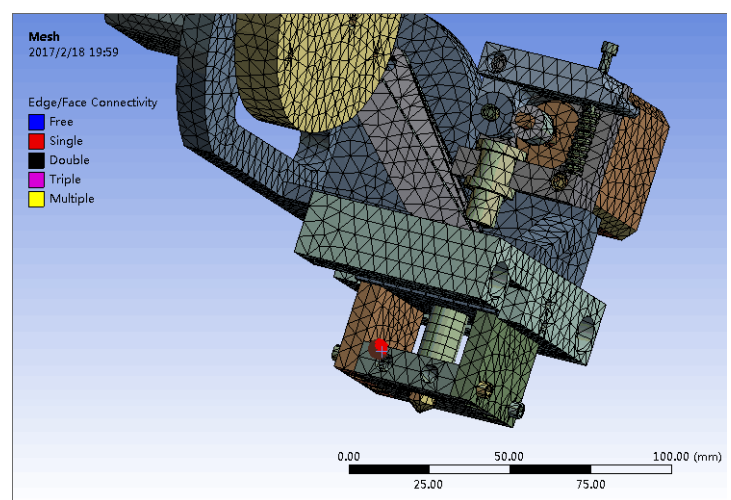

Figure 3.4 finite element mesh

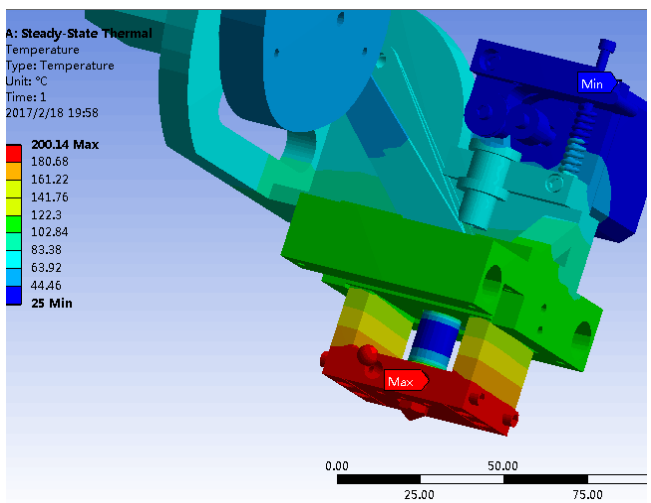

Figure 3.5 nozzle temperature field distribution

From the simulation results, the temperature of the heating nozzle is about 200 degrees. Although the design requirements have been met, the temperature of the moving block is 110 degrees. The high temperature of the moving bolock will affect the printing accuracy and equipment service life. Therefore, it is necessary to optimize the device to reduce the heat transfer from the nozzle to the extrusion device. So, the heat conduction process is reduced by using the heat insulation gasket between the fixing frame of the heating head and the fixing bracket of the printing device. Also, the nozzle fixing frame structure is optimized. The optimized mesh is shown in 3.6 and the simulation result is shown in 3.7 .
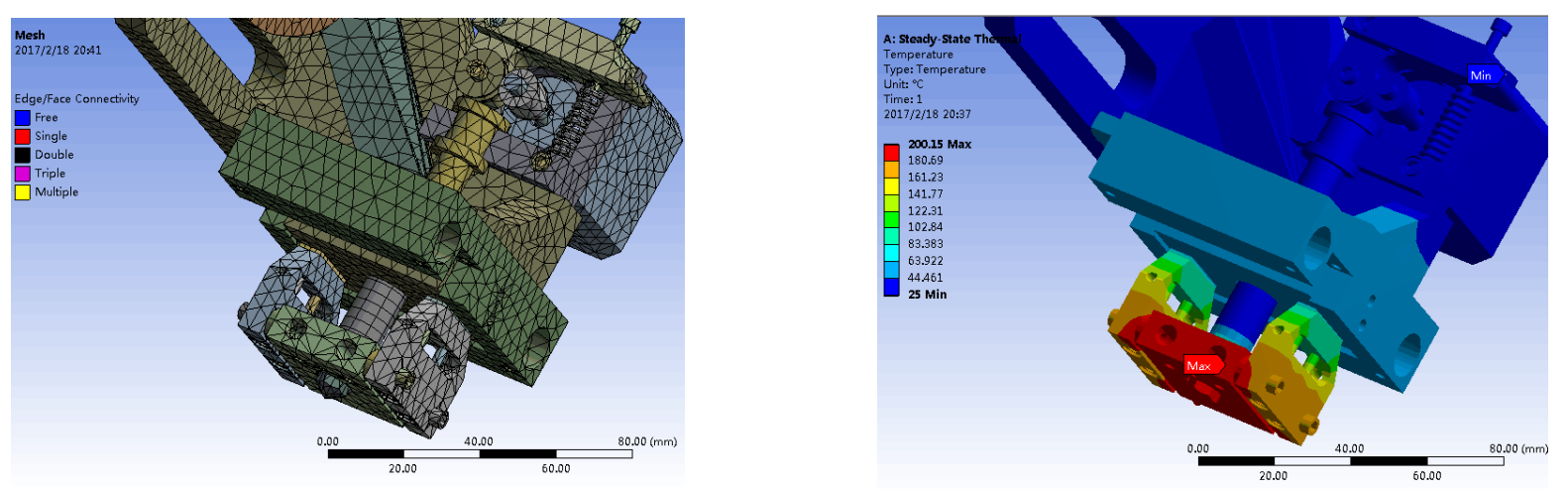

Figure 3.6 optimized finite element mesh Figure 3.7 temperature field with heat insulation gasket with heat insulation gasket

From the temperature field distribution in Figure 3.7, it can be seen that the temperature of nozzle and heating block are still high, which is about 200 degrees centigrade. But the temperature of the moving block has been reduced by 60 degrees, and it is about 40 degrees. So, the optimized extrusion nozzle device has a reasonable temperature distribution to meet the continuous carbon fiber 3D printing.

Thermal equilibrium simulation. In the nozzle system, the two heating rods parameters are: rated voltage $12 \mathrm{~V}$, rated power $40 \mathrm{~W}$. The thermal energy is provided in this manner. The air is cooled by means of heat convection. The wind speed is $5 \mathrm{~m} / \mathrm{s}$ and the coefficient is 100 . The nozzle, fixed frame, heating block and motor surface are selected as the radiation surface. The radiation coefficient is 0.3. The thermal equilibrium temperature field is shown in Figure 3.8. 


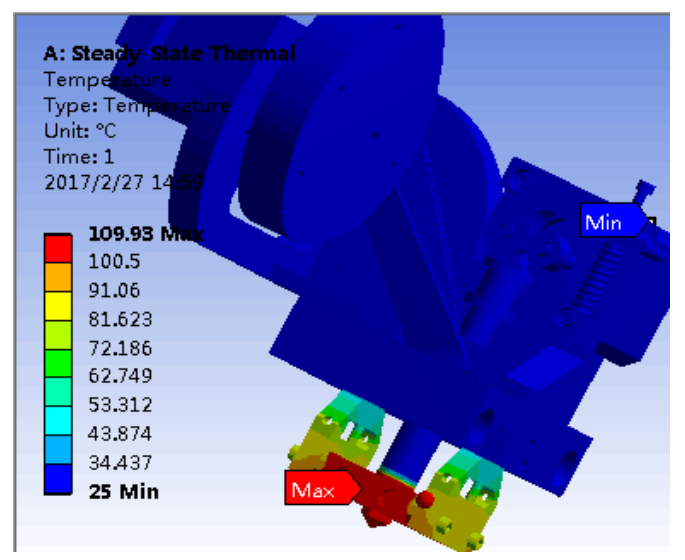

Figure 3.8 Thermal equilibrium simulation

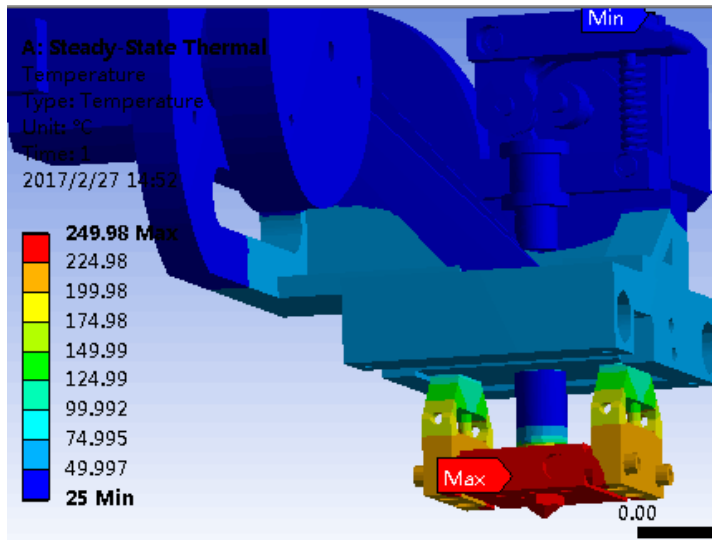

Figure 3.9 Thermal equilibrium with insulation cotton

From the simulation result in the thermal equilibrium state, the nozzle temperature is only 109.9 degrees which can't meet the requirements of the 200-210 degrees to make the base material reach the melting state. So, it is necessary to optimize the nozzle device further. In the printing nozzle system, the heating rod and the heating block are the heat sources, so, it is necessary to minimize the heat loss in these two parts. Therefore, the upper surface of the heating block is insulated by insulation cotton to improve the temperature. The improved simulation results are shown in Figure 3.9. From the result, the heat dissipation effect of the heating block is reduced obviously. The maximum temperature of nozzle and heating block in the equilibrium state can reach 249.9 degrees.

\section{Realization and experiment of 3D printing nozzle}

Based on the simulation result here in before, the 3D printing nozzle structure of continuous carbon fiber composite is shown in Figure 3.10. First, the heat insulation gasket is used between the nozzle fixing frame and the device fixing frame, also the nozzle fixing frame structure is optimized. In addition, the upper surface of the heating block is insulated by the heat insulation cotton to prevent the heat loss.
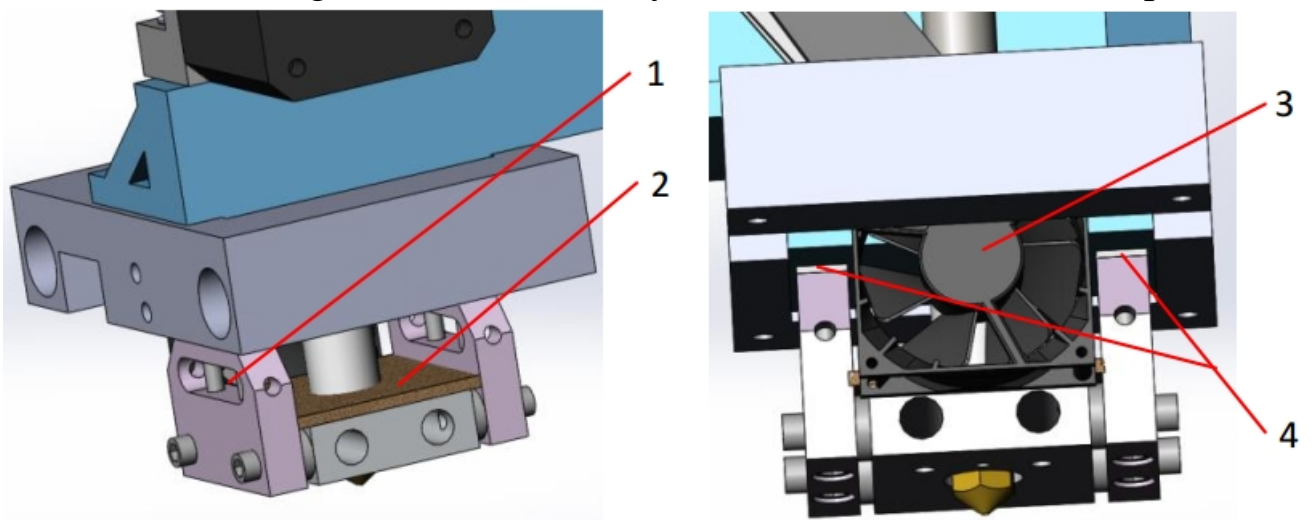

Figure 3.10 model of the nozzle system

1.nozzle fixing frame, 2.heat insulation cotton, 3.heat dissipation fan, 4.heat insulation gasket

Experimental study of the continuous carbon fiber 3D printing is shown in Figure 3.11 and 3.12. First, the nozzle temperature is set as 200 degrees. The temperature sensor's feedback is detected by the control system. Also, the non contact temperature sensor is used to measure the temperature of different parts. The result shows that the nozzle system has a good temperature field distribution for continuous carbon fiber 3D printing. The 3D printing model of continuous carbon fiber is shown in Figure 3.13. 


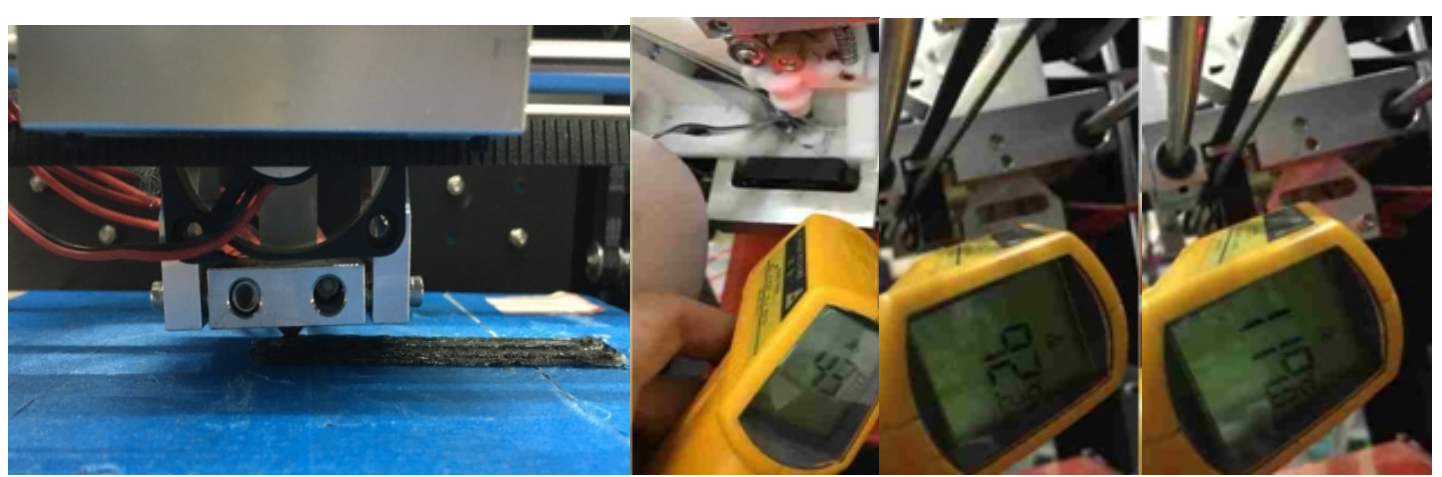

Figure 3.11 3D printing nozzle and the emperature of movement slider, upper part of the nozzle fixed block and lower part of the nozzle fixed block

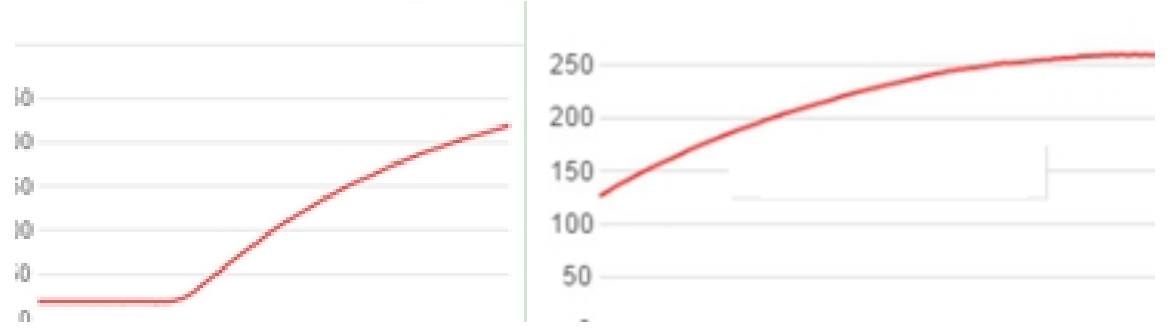

Figure 3.12 the temperature curve of nozzle heating process and thermal equilibrium state

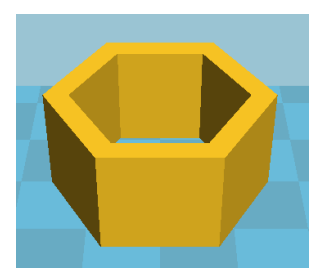

(1)hexagonal 3D model

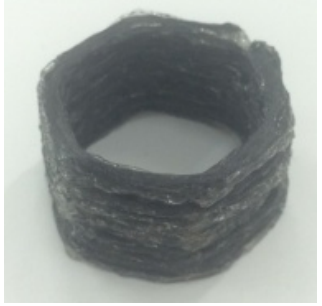

(c) hexagonal 3D printing model

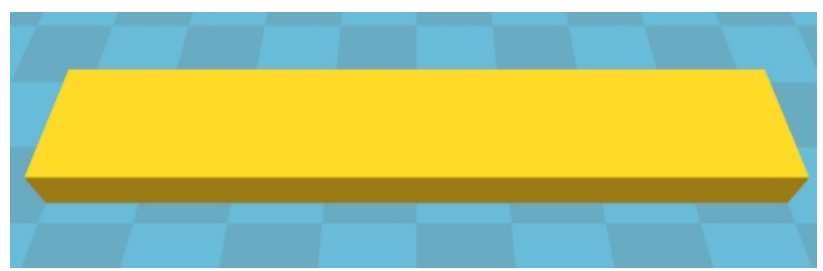

(b) standard prototype model for pressure test

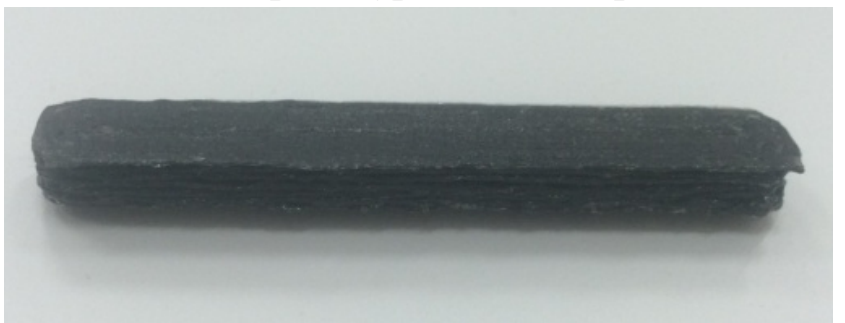

(d) prototype model of seven layers

Figure 3.13 3D printing model of continuous carbon fiber

\section{Conclusion}

Because of the continuity character of the carbon fiber composite, the reasonable nozzle structure determines the forming quality. The continuous carbon fiber 3D printing needs a high temperature in a short time for rapid melting; meanwhile, it needs to avoid the nozzle blocked problem caused by the front end material softening due to the multiple heat dissipation. In this paper, according to the characters of the continuous carbon fiber composite, the nozzle heating and cooling system are designed. Based on the temperature field thermodynamic analysis, the relationship between the nozzle structure and the temperature distribution is revealed. Also, the nozzle structure is optimized. The local temperature is stable at about 200 degrees and the heat dissipation effect is normal. It is proved by experiments that the requirement of continuous carbon fiber 3D printing based on fused deposition modeling is satisfied. 


\section{Acknowledgements}

This work was financially supported by the Fundamental Research Funds for the Central Universities(WUT: 2017II26GX) and the China's Post-doctoral Science Fund(2016M602380).

\section{References}

[1] Wei G, Zhang Y, Ramanujan D, et al. The status, challenges, and future of additive manufacturing in engineering[J]. Computer-Aided Design, 2015, 69:65-89.

[2] Tekinalp H L, Kunc V, Velez-Garcia G M, et al. Highly oriented carbon fiber-polymer composites via additive manufacturing[J]. Composites Science \& Technology, 2014, 105:144-150.

[3] S.Kumar, J.-P Kruth. Composites by rapid prototyping technology[J]. Materials and Design, 2010,31(2):850-856.

[4] Macdonald E, Salas R, Espalin D, et al. 3D printing for the rapid prototyping of structural electronics [J]. Dissertations \& Theses - Gradworks, 2013,2:234-242.

[5] Ken-ichiro Mori, Tomoyoshi Maeno, Yuki Nakagawa. Dieless forming of carbon fibre reinforced plastic parts using 3D printer[J]. Procedia engineering, 2014,81:1595-1600.

[6] Tian X, Liu T, Yang C, et al. Interface and performance of 3D printed continuous carbon fiber reinforced PLA composites[J]. Composites Part A Applied Science \& Manufacturing, 2016, 88:198-205.

[7] Li N, Li Y, Liu S. Rapid prototyping of continuous carbon fiber reinforced polylactic acid composites by 3D printing[J]. Journal of Materials Processing Technology, 2016, 238:218-225.

[8] Melenka G W, Cheung B K O, Schofield J S, et al. Evaluation and prediction of the tensile properties of continuous fiber-reinforced 3D printed structures[J]. Composite Structures, 2016, 153:866-875. 